\title{
Coherent pumping of a Mott insulator: Fermi golden rule versus Rabi oscillations
}

\author{
F. Hassler ${ }^{1}$ and S. D. Huber ${ }^{2,1}$ \\ ${ }^{1}$ Institute for Theoretical Physics, ETH Zurich, 8093 Zurich, Switzerland \\ ${ }^{2}$ Department of Condensed Matter Physics, The Weizmann Institute of Science, Rehovot, 76100, Israel
}

(Dated: November 6, 2018)

\begin{abstract}
Cold atoms provide a unique arena to study many-body systems far from equilibrium. Furthermore, novel phases in cold atom systems are conveniently investigated by dynamical probes pushing the system out of equilibrium. Here, we discuss the pumping of doubly-occupied sites in a fermionic Mott insulator by a periodic modulation of the hopping amplitude. We show that deep in the insulating phase the many-body system can be mapped onto an effective two-level system which performs coherent Rabi oscillations due to the driving. Coupling of the two-level system to the remaining degrees of freedom renders the Rabi oscillations damped. We compare this scheme to an alternative description where the particles are incoherently pumped into a broad continuum.
\end{abstract}

PACS numbers: 03.75.Ss, 71.10.Fd, 31.15.aq

Cold atoms in optical lattices provided us with numerous insights into the paradigmatic quantum phase transition displayed by interacting lattice bosons [1, 2]. Progress in the same setup with fermionic atoms has now led to new, equally-exciting results in the study of the Hubbard model [3, 4, 5, 6, 7]. A decrease of the compressibility when crossing over from the weakly to the strongly interacting region was reported [8, 9]. Furthermore, in the experiment by Jördens et al. [8], the strongly correlated regime of fermions in an optical lattice has been investigated by harmonically modulating the strength of the light beams forming the optical lattice. The modulation of the lattice generates doubly-occupied sites (doublons). This generation of doublons deep in the Mott insulator, where the interaction $U$ dominates over the hopping $t_{0}$, is the focus of our present work. Experimentally, the fraction of particles on doubly-occupied sites (or double occupancy $\mathcal{D}$ ) was measured after modulating the optical lattice. For $U / 6 t_{0}=13.6$, a saturation was observed at around $\mathcal{D}_{\text {sat }}=30 \%$ after a modulation time $\tau_{\text {mod }} \approx 50 h / U$ (50 "cycles") [8] which sets an upper bound on the saturation time $\tau_{\text {sat }} \leq \tau_{\bmod }$ [see Fig. [1(a)].

We consider fermionic atoms, such as ${ }^{40} \mathrm{~K}$, subject to an optical lattice in the framework of the Hubbard model

$$
H=-t_{0} \sum_{\langle i, j\rangle, \sigma}\left(c_{i \sigma}^{\dagger} c_{j \sigma}+\text { H.c. }\right)+U \sum_{i} c_{i \uparrow}^{\dagger} c_{i \uparrow} c_{i \downarrow}^{\dagger} c_{i \downarrow},
$$

at half filling and with the interaction $U$ dominating over the hopping amplitude $t_{0}$. Here, the fermionic operators $c_{i \sigma}^{\dagger}$ create an atom in one of two possible hyperfine states $\sigma=\uparrow, \downarrow$ at site $i$ and the sum $\langle i, j\rangle$ runs over nearest neighbors. The ground state of the system consists of one particle per site with the hopping $t_{0}$ suppressed due to the strong on-site repulsion $U$. As the temperature $T$ in the experiment is larger than the super-exchange coupling $J=4 t_{0}^{2} / U$ [8], no significant quantum correlations over the bonds are present and the ground state of the system is largely composed of singly-occupied sites $(T \ll U)$ with a random spin-direction on each site. A harmonic modulation of the lattice with frequency $\omega \approx U / \hbar$ transfers
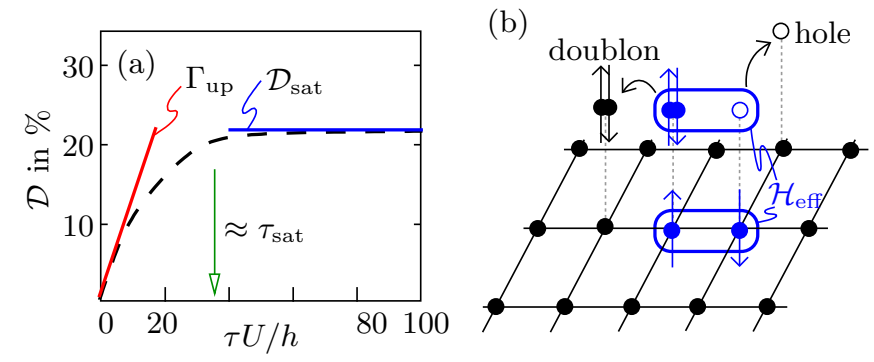

FIG. 1: (color online) (a) Sketch of an experimental sequence for the generation of double occupancy by modulating the optical lattice during a time $\tau$. After an initial buildup of doubly-occupied sites with a rate $\Gamma_{\text {up }}$, the fraction of particles residing in doubly-occupied sites saturates at a value $\mathcal{D}_{\text {sat }}$ after a time $\tau_{\text {sat }}$. (b) Illustration of the effective Hilbert space $\mathcal{H}_{\text {eff }}$ consisting of one bond embedded in an array of sites. The bath degrees of freedom consist of all doublon-hole configurations in which the pair resides on a different bond than where it was created.

energy to the system and (possibly) promotes a particle across the bond, thereby creating a doubly-occupied site next to an empty site, a doublon-hole pair.

Despite the fact that the Hubbard model represents a many-body problem in a huge Hilbert space, we adopt a simple description via modeling a single (typical) bond [Fig. 1(b)], and taking the remaining bonds into account as an effective bath. Two different ideas lend themselves to modeling the process of pumping doublon-hole pairs [see Fig. 2]: (a) the direct excitation of the bond via incoherent transitions into the doublon-hole continuum with a rate given by the Fermi golden rule

$$
\Gamma_{\bmod }^{\mathrm{FGR}}=\frac{2 \pi}{\hbar}\left|\left\langle f\left|H_{\mathrm{mod}}\right| g\right\rangle\right|^{2} \rho\left(E_{g}+\hbar \omega\right) ;
$$

here, $E_{g}$ denotes the energy of the ground state, $\rho$ is the density of states of the doublon-hole continuum, and $\left\langle f\left|H_{\text {mod }}\right| g\right\rangle$ is the matrix element arising from the modulation of the lattice. Taking the modulation strength to be given by $\delta t$ and estimating the density of states 
(a)

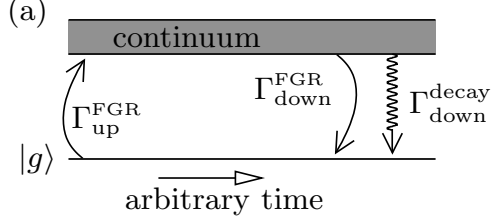

(b)

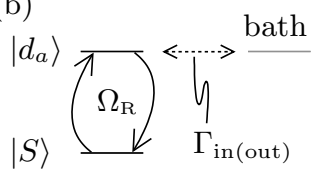

FIG. 2: Comparison of two possible descriptions: (a) A rate equation description where an incoherent pump rate $\left[\Gamma_{\mathrm{up}}^{\mathrm{FGR}}\right]$ into a broad doublon-hole continuum and subsequent decay $\left[\Gamma_{\text {down }}^{\text {decay }}\right]$ or incoherent stimulated emission $\left[\Gamma_{\text {up }}^{\mathrm{FGR}}\right]$ lead to a saturation. (b) Coherent Rabi oscillations [with frequency $\Omega_{R}$ ] between the ground state and a single exited state where decay out of the effective Hilbert space is introduced via coupling to a bath $\left[\Gamma_{\text {in (out) }}\right]$.

as $\rho \propto 1 / t_{0}$, we obtain the scaling of the pump rate $\Gamma_{\bmod }^{\mathrm{FGR}} \propto \delta t^{2} / t_{0}$ as a function of the hopping amplitude $t_{0}$ and the strength of the modulation $\delta t$. (b) Coherent Rabi oscillations in the isolated bond between the ground state of the bond and the doubly-occupied excited state with a Rabi frequency $\Omega_{\mathrm{R}} \propto \delta t$ proportional to the modulation strength. The Rabi oscillations generate doublon-hole pairs and lead to a periodic modulation (with frequency $\Omega_{\mathrm{R}}$ ) of the double occupancy. Note that the rates of the two (extreme) processes, incoherent pumping into a band (a) (with rate $\Gamma_{\bmod }^{\mathrm{FGR}}$ ) and coherent excitation of doublon-hole pairs (b) (with rate $\hbar \Omega_{R}$ ), have a different dependence on the modulation strength $\delta t$ and can therefore be discriminated experimentally.

Here, we propose a third way where the coherent Rabi oscillations produce a doublon-hole pair and, subsequently, hopping of the doublon (or the hole) to the neighboring sites leads to an incoherent decay of the excitation into the doublon-hole continuum. The process is governed by two time scales, the Rabi frequency $\Omega_{\mathrm{R}} \propto \delta t$ which periodically modulates the double occupancy and the decay $\Gamma_{\text {hop }} \propto t_{0}$ which leads to a saturation.

A similar system, where a two-level atom is coupled simultaneously to a laser field and to vacuum modes of the radiation field, is well-studied in quantum optics [10]. The two-level atom corresponds to the two states on the bond and the laser plays the role of the harmonic modulation of the lattice. However, in the atomic example, the spontaneously emitted photon takes the atom back into the ground state where it continues with the Rabi cycles, while in the present case the doublon (or hole) itself exits the system. The present process then resembles more strongly that of optical pumping into a third nondecaying level, with the accumulation in the pumped level playing the role of the buildup in double occupancy.

In the following, we first describe the effective model in the single bond Hilbert space driven by a harmonic modulation of the lattice and derive the frequency of the Rabi oscillations. We then account for the bath consisting of the surrounding sites via a master equation approach and analyze it in different limits. We compare this approach to the situation where the bond is directly excited into the doublon-hole continuum; see (a) above. It turns out that the time scale $\left(\tau_{\mathrm{R}}\right)$ for the Rabi cycle is in rough agreement with the experimental value of the saturation time, whereas the treatment via a direct excitation into the doublon-hole continuum leads to a time scale $\left(\tau_{\mathrm{FGR}}\right)$ which is an order of magnitude larger $\left(\tau_{\mathrm{sat}}^{\exp } \lesssim \tau_{\mathrm{R}} \ll \tau_{\mathrm{FGR}}\right)$. For a quantitative agreement, one has to go beyond a single bond in the description of the pumping process. Different bonds are coupled via the pumping field, leading to a Dicke-like description [11], but, most likely, manybody effects dominate the coupling of different bonds; a systematic study of larger clusters deserves future efforts.

In deriving the effective Hamiltonian governing the dynamics in the Hilbert space of a single bond (the sites are labeled by $\mathrm{L}$ and $\mathrm{R}$ ), we neglect the confining potential and focus on a homogeneous three dimensional setup. The ground state at half-filling involves one particle per site without any spin order, i.e., the particles on the bond occupy the three triplet states $\left|T^{ \pm 1}\right\rangle=c_{\mathrm{L} \uparrow(\downarrow)}^{\dagger} c_{\mathrm{R} \uparrow(\downarrow)}^{\dagger}|0\rangle, \quad\left|T^{0}\right\rangle=\frac{1}{\sqrt{2}}\left(c_{\mathrm{L} \uparrow}^{\dagger} c_{\mathrm{R} \downarrow}^{\dagger}+c_{\mathrm{L} \uparrow}^{\dagger} c_{\mathrm{R} \downarrow}^{\dagger}\right)|0\rangle$, and the singlet state $|S\rangle=\frac{1}{\sqrt{2}}\left(c_{\mathrm{L} \uparrow}^{\dagger} c_{\mathrm{R} \downarrow}^{\dagger}-c_{\mathrm{L} \uparrow}^{\dagger} c_{\mathrm{R} \downarrow}^{\dagger}\right)|0\rangle$. These states constitute the four degenerate ground states of a single bond and are realized with equal probability of $1 / 4$ each. A harmonic modulation of the lattice beam with $V=V_{0}+\delta V \cos (\omega \tau)$ leads to a time-dependent hopping Hamiltonian

$$
H_{\mathrm{hop}}=\delta t \cos (\omega \tau) \sum_{\sigma}\left(c_{\mathrm{L} \sigma}^{\dagger} c_{\mathrm{R} \sigma}+\text { H.c. }\right),
$$

with $\delta t / t_{0}=\left(3 / 4-\sqrt{V_{0} / E_{r}}\right) \delta V / V_{0}$ and $\tau$ denoting the time. This expression is valid for $\delta V / V_{0} \ll 1$ and $\delta V / V_{0} \ll \sqrt{E_{r} / V_{0}}[12$. For current experiments with fermions [8], where $\delta V / V_{0}=0.1$ and $V_{0}$ does not exceed $10 E_{r}$, both conditions are safely fulfilled. In the derivation of Eq. (3), we have dropped the constant hopping amplitude proportional to $t_{0}$, as hopping is blocked in the Mott insulator at half-filling. The hopping amplitude $t_{0}$ will be reintroduced later as it is responsible for the decay of the doublon-hole pair by coupling the bond to the surrounding lattice. The modulation couples the ground states to the doublon-hole states $\left|d_{\mathrm{L}(\mathrm{R})}\right\rangle=c_{\mathrm{L}(\mathrm{R}) \uparrow}^{\dagger} c_{\mathrm{L}(\mathrm{R}) \downarrow}^{\dagger}|0\rangle$, with the doublon located on the left (right) site. These states exhibit an energy offset $U$ with respect to the energy of the ground states. The total Hamiltonian in the Hilbert space spanned by the four ground states (triplet and singlet) and the two doublon-hole pairs is given by

$$
\begin{array}{r}
H_{\mathrm{eff}}=\sum_{\alpha=\mathrm{L}, \mathrm{R}} U\left|d_{\alpha}\right\rangle\left\langle d_{\alpha}\left|+H_{\mathrm{hop}} \approx \sum_{\alpha=\mathrm{L}, \mathrm{R}} U\right| d_{\alpha}\right\rangle\left\langle d_{\alpha}\right| \\
+\delta t\left[e^{i \omega \tau / \hbar}\left|d_{\mathrm{a}}\right\rangle\left\langle S\left|+e^{-i \omega \tau / \hbar}\right| S\right\rangle\left\langle d_{\mathrm{a}}\right|\right]
\end{array}
$$

where the first term describes the energy offset by $U$ and the second term the driving due to the lattice modulation (3). In going from the first to the second equation in (41), the rotating wave approximation has been used and we have introduced the active and passive doublonhole states given by $\left|d_{\mathrm{a}(\mathrm{p})}\right\rangle=\left(\left|d_{\mathrm{L}}\right\rangle \pm\left|d_{\mathrm{R}}\right\rangle\right) / \sqrt{2}$; these are 
related to the left (right) doublon-hole state via a basis change. Driving the system on resonance with $\hbar \omega=U$, the singlet state is coupled to the excited state via Rabi oscillations. Within the present description, off-resonant coupling can be described in the standard manner. As can be seen from Eq. (4), the triplet states are unaffected by the lattice modulations. Only the singlet state is coupled to the active doublon-hole pairs $\left|d_{\mathrm{a}}\right\rangle$. Going over to the interaction representation with the reference dynamics given by the first term in (4), the relevant Hamiltonian in the effective two-level system $\mathcal{H}_{\text {eff }}=\operatorname{span}\left\{\left|d_{\mathrm{a}}\right\rangle,|S\rangle\right\}$ can be expressed as a matrix

$$
\mathbf{H}=\left(\begin{array}{cc}
0 & \delta t \\
\delta t & 0
\end{array}\right)
$$

which describes the coherent oscillations in the two-level system with the Rabi frequency

$$
\Omega_{\mathrm{R}}=\frac{2 \delta t}{\hbar} .
$$

The triplet states and the passive doublon-hole pair remain eigenstates of the time-dependent Hamiltonian and therefore particles in such states are not driven.

So far, we have neglected the effect of $t_{0}$ on the excited state $\left|d_{\mathrm{a}}\right\rangle$. Provided the bond is in a doublon-hole state, we can lose the state from our single bond description via the hopping of the doublon (or the hole) out of the bond; cf. Fig. 11(b) [13]. This effect is captured by a bath representing the states with the doublon and hole separated from each other. Note that the bath states still contribute to the double occupancy (as there is still a doublon present) but do not participate in Rabi oscillations (as the doublon and the hole are not located on the same bond). We denote the rate at which the doublonhole bond decays into a separated doublon and a hole by $\Gamma_{\text {out }}$, and the reverse rate at which a doublon and a hole collide to form a doublon-hole pair by $\Gamma_{\text {in }}$. Taking the coupling to the bath into account and performing a standard analysis similar to 14] leads to an incoherent term (besides the standard term proportional to $[\mathrm{H}, \rho]$ ) in the evolution of the reduced density matrix $\rho$ in the effective Hilbert space $\mathcal{H}_{\text {eff }}=\operatorname{span}\left\{\left|d_{\mathrm{a}}\right\rangle,|S\rangle\right\}$ of the form

$$
\left.\frac{\partial \rho}{\partial \tau}\right|_{\text {inc }}=-\frac{1}{2}\left(\begin{array}{cc}
2\left(\Gamma_{\text {out }} \rho_{11}-\Gamma_{\text {in }} \rho_{0}\right) & \Gamma_{\text {out }} \rho_{12} \\
\Gamma_{\text {out }} \rho_{21} & 0
\end{array}\right) .
$$

Different from a standard master equation [14], Eq. (7) is not probability preserving, since with probability $\rho_{0}=$ $1-\rho_{11}-\rho_{22}$ the doublon and the hole have separated and thus have left the effective Hilbert space $\mathcal{H}_{\text {eff. }}$ In the steady state situation with $\partial_{\tau} \rho=0$, the probability that the system contributes to the double occupancy is given by

$$
\rho_{\mathrm{D}}^{\mathrm{sat}}=\rho_{0}^{\mathrm{sat}}+\rho_{11}^{\mathrm{sat}}=1-\rho_{22}^{\mathrm{sat}}=\frac{\Gamma_{\mathrm{out}}+\Gamma_{\mathrm{in}}}{\Gamma_{\mathrm{out}}+2 \Gamma_{\mathrm{in}}},
$$

the sum of the probabilities that the system is in the doublon-hole pair state $\left(\rho_{11}\right)$ or in the bath $\left(\rho_{0}\right)$. Note

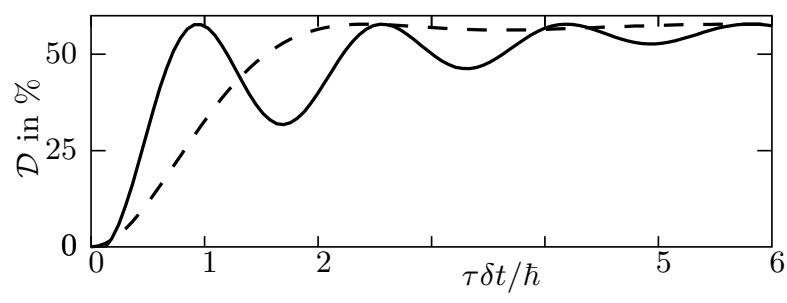

FIG. 3: Fraction of particles residing in doubly-occupied sites after modulation time $\tau$. After a buildup time corresponding to approximately one $\pi$-pulse $\left[\tau_{\mathrm{R}}=\pi / \Omega_{\mathrm{R}}=\hbar \pi / 2 \delta t\right]$, the decay, with (weak) strength $\Gamma_{\text {out }}=\delta t / \hbar \approx 0.24 t_{0} / \hbar$ and $\Gamma_{\text {in }}=$ 0 , leads to a saturation at $\mathcal{D}_{\text {sat }}=58 \%$ corresponding to the fact that all "active" bonds, singlets, are excited in the system (solid line). A more realistic situation where the damping is increased by a factor of 2 renders the oscillations barely visible (dashed line). Taking into account a finite value of $\Gamma_{\text {in }}$ leads to a decrease of the saturation value. Note that, irrespective of the saturation value, the time scale needed to pump the system into a doubly occupied state is given by the Rabi frequency.

that atoms which left the effective Hilbert space still contribute to the double occupancy. The bath can be pictured as a gas of doublons and holes (with equal numbers). Initially, the bath does not contain any particles such that $\Gamma_{\text {in }}=0$ (this approximation remains valid as long as the density of particle is low); furthermore, we estimate the escape rate by $\Gamma_{\text {out }}=\Gamma_{\text {hop }} \sim t_{0} / \hbar$. For $\Gamma_{\text {in }}=0$, Eq. (8) provides the saturation value $\rho_{\mathrm{D}}^{\text {sat }}=1$, i.e., the singlet is certainly transferred to one of the doublon-hole states. Using the dilute gas approximation, $\Gamma_{\mathrm{in}}=0$, we can solve the equation of motion for the density matrix

$$
\partial_{\tau} \rho=\frac{1}{i \hbar}[\mathrm{H}, \rho]-\frac{\Gamma_{\text {out }}}{2}\left(\begin{array}{cc}
2 \rho_{11} & \rho_{12} \\
\rho_{21} & 0
\end{array}\right) .
$$

Assuming that initially the bond is in a singlet state

$$
\rho(\tau=0)=\left(\begin{array}{ll}
0 & 0 \\
0 & 1
\end{array}\right)
$$

Eq. (9) gives the time evolution of the density matrix of the bond. Qualitatively, the weight is transferred to the exited state $\left|d_{\mathrm{a}}\right\rangle$ at a rate $\hbar \Omega_{\mathrm{R}}$ and then the decay into other states occurs with a rate $\Gamma_{\text {out }}$ (cf. Fig. 3).

To calculate the fraction of particles in a doublon state, we need to know how many bonds are initially in a singlet configuration. We estimate the probability that a specific site is part of a singlet as $P_{S}=1-(3 / 4)^{d} \approx 0.58$, here $d=$ 3 is the "dimension" of the lattice modulation (it counts the number of bonds per site) and we have assumed that the singlets are independent of each other and occupied with a probability $1 / 4$. The fraction of particles in a doublon state is given by

$$
\mathcal{D}=P_{S} \rho_{\mathrm{D}}(\tau)
$$

with $\rho_{\mathrm{D}}(\tau)=1-\rho_{22}(\tau)$ the probability that this singlet is transferred to a doublon state. The system quickly 
reaches a steady state with $\mathcal{D}_{\text {sat }}=P_{S}$ (cf. Fig. 3). In three dimensions, we reach a maximal value $\mathcal{D}_{\text {sat }}=58 \%$. Note that at this value of double occupancy the dilute gas approximation $\Gamma_{\mathrm{in}}=0$ becomes invalid. We expect that $\Gamma_{\text {in }}$ increases for increasing double occupancy and, therefore, the double occupancy saturates at a lower value. A theory that aims at a quantitative result for the saturation value should take such effects into account and solve the system self-consistently. Assuming that for long times the density of particles in the bath is high such that $\Gamma_{\text {in }} \gg \Gamma_{\text {out }}$, we obtain the result that $\rho_{\mathrm{D}}^{\text {sat }}=50 \%$ [cf. Eq. (8)] and $\mathcal{D}_{\text {sat }}=29 \%$, comparable to experimental results [8]. The time scale needed to excite the system is approximately given by the time $\tau_{\mathrm{R}}=\pi / \Omega_{\mathrm{R}}$ of a $\pi$ pulse, irrespective of the saturation value obtained. Note that the finite line-width of the perturbing laser, the scattering between doublons, and an inhomogeneous environment for a single bond due to the trap are other processes which lead to a dephasing of the coherent single bond dynamics. As the effect of $t_{0}$ on the excited state leads already to an over-damped dynamics, these other effects are neglected here.

Another approach to describe the saturation in the double occupancy is based on a rate equation for the doublon fraction. The rates at which excitations are generated are taken into account via a rate equation for the doublon concentration

$$
\partial_{\tau} \mathcal{D}=(1-\mathcal{D}) \Gamma_{\text {up }}-\mathcal{D} \Gamma_{\text {down }} .
$$

Within this description two independent processes are assumed. First, the system is excited with a rate $\Gamma_{\text {up }}$. Second, at a later uncorrelated stage, these excitations are removed with a rate $\Gamma_{\text {down }}$ [cf. Fig. 2(a)]. In order to compare the two schemes, the rate equation and the Rabi oscillation, we extract the time scales of both processes. The Fermi golden rule matrix element is approximately given by $\delta t^{2}[$ []. Additionally, we need an estimate for the density of states. Once excited, the doublon as well as the hole can move freely through the lattice. The corre- sponding doublon-hole continuum acquires a width of approximately $24 t_{0}$ (in three dimensions). A crude estimate for the density of states is then given by $\rho \approx 1 / 24 t_{0}$. We then arrive at the two time scales $\tau_{\mathrm{R}}=\pi / \Omega_{\mathrm{R}}=\pi \hbar / 2 \delta t$ and $\tau_{\mathrm{FGR}}=(\hbar / 2 \pi) 24 t_{0} / \delta t^{2}=\left(24 t_{0} / \pi^{2} \delta t\right) \tau_{\mathrm{R}} \approx 10 \tau_{\mathrm{R}}$, associated with a Rabi oscillation and the rate equation, respectively. For the last step, we have used $\delta t / t_{0} \approx 0.24$ corresponding to current experiments. Note that the time scale to reach saturation in the incoherent Fermi golden rule approach is an order of magnitude larger than the one predicted by the Rabi oscillation picture. In Ref. [8] the saturation sets in before 50 "cycles", which is even smaller than $\tau_{\mathrm{R}}\left[\tau_{\bmod }=50 h / U=(200 \delta t / U) \tau_{\mathrm{R}} \approx\right.$ $0.6 \tau_{\mathrm{R}}$ for $U / 6 t_{0}=13.6$ and $\left.\delta t / t_{0} \approx 0.24\right]$, promoting the picture of the coherent Rabi oscillations. In an experiment, one can test one theory against the other by changing the coupling strength $\delta t$ while keeping all other parameters, such as the bandwidth $1 / 24 t_{0}$, constant. The time scale for the saturation scales as $\delta t^{-2}$ in the Fermi golden rule picture, whereas Rabi oscillations produce a saturation time inversely proportional to $\delta t$.

In summary, we have discussed two alternative explanations for the buildup and the saturation of the double occupancy in the modulation experiments of Ref. [8] deep in the Mott insulating phase. The comparison to experiment favors the description in terms of an effective two-level system with coherent driving, leading to the characteristic time scale of $\tau_{\mathrm{R}} \approx \pi / \Omega_{\mathrm{R}}=\hbar \pi / 2 \delta t$ for half a Rabi cycle. Given the large decay rate proportional to $t_{0}>\hbar \Omega_{\mathrm{R}} \approx \delta t$, only one $\pi$-pulse is observable. The predictions can be experimentally tested by studying the dependence of the saturation time on various system parameters as $V_{0} / E_{r}, \delta V / V_{0}$, and $t_{0} / U$.

We acknowledge fruitful discussions with E. Altman, G. Blatter, H. P. Büchler, the group of T. Esslinger, S. Fölling, L. Goren, and A. Rüegg. This work was supported by the Swiss National Foundation through the NCCR MaNEP.
[1] M.P.A. Fisher, P.B. Weichman, G. Grinstein, and D.S. Fisher, Phys. Rev. B 40, 546 (1989).

[2] M. Greiner, O. Mandel, T. Esslinger, T.W. Hänsch, and I. Bloch, Nature 415, 39 (2002).

[3] V.W. Scarola, L. Pollet, J. Oitmaa, and M. Troyer, arXiv:0809.3239 (2008).

[4] L. De Leo, C. Kollath, A. Georges, M. Ferrero, and O. Parcollet, Phys. Rev. Lett. 101, 210403 (2008).

[5] R.W. Helmes, T.A. Costi, and A. Rosch, Phys. Rev. Lett. 100, 056403 (2008).

[6] S.D. Huber and A. Rüegg, Phys. Rev. Lett. 102, 065301 (2009).

[7] C. Kollath, A. Iucci, I.P. McCulloch, and T. Giamarchi, Phys. Rev. A 74, 041604(R) (2006).

[8] R. Jördens, N. Strohmaier, K. Günter, H. Moritz, and T. Esslinger, Nature 455, 204 (2008).

[9] U. Schneider, L. Hackermuller, S. Will, T. Best, I. Bloch,
T.A. Costi, R.W. Helmes, D. Rasch, and A. Rosch, Science 322, 1520 (2008).

[10] C. Cohen-Tannoudji, in Frontiers in Laser Spectroscopy, edited by R. Balian, S. Haroche, and S. Liberman, Proceedings of the Les Houches Summer School of Theoretical Physics, 1975 (North-Holland, Amsterdam, 1977).

[11] R.H. Dicke, Phys. Rev. 93, 99 (1954).

[12] When deriving (3), care has to be taken in expanding the exponential part of the hopping amplitude [15].

[13] We think about the singly-occupied lattice as being the vacuum with excitations given by doublons and holes.

[14] C.W. Gardiner and P. Zoller, Quantum Noise, chapter 5.1 (Springer, Berlin, 2000).

[15] W. Zwerger, J. Opt. B: Quantum Semiclassical Opt. 5, S9 (2003). 